\title{
Meter without rhythmic pattern repetitions increases pre-attentive processing
}

\author{
Rui Gomes ${ }^{\mathrm{a}}$, Regina Abreu ${ }^{\mathrm{ab}}$, and Susana Silva ${ }^{\mathrm{a}}$
}

${ }^{a}$ Center for Psychology at University of Porto, University of Porto, Porto, Portugal

${ }^{b}$ Norwegian University of Science and Technology, Trondheim, Norway

Word count (body text): 7963

Corresponding author:

Susana Silva

Rua Alfredo Allen, s/n, 4200-135, Porto, Portugal

susanamsilva@fpce.up.pt

+351964366725

Orcid Id: 0000-0003-2240-1828 
METER WITHOUT RHYTHMIC PATTERN REPETITIONS INCREASES PRE-ATTENTIVE PROCESSING

\begin{abstract}
Processing musical meter - the organization of time into regular cycles of strong and weak beats - requires abstraction from the varying rhythmic surface. Several studies investigated whether meter processing requires attention, or if it can be both pre-attentive and attentive. While findings on temporal expectation (processing meter per se) indicated benefits of attention, studies on meter processing in a more complex, dual-task context (meter used for temporal orientation) consistently reported pre-attentive processing. Also, while surface-based approaches to meter (meter aided by pattern repetition) showed some benefits of attention, structural approaches (meter not aided by pattern repetition, increased complexity) found pre-attentive-only processing. Therefore, in the present study we hypothesized that pre-attentive processing increases with cognitive load, and we compared surface with structural meter processing. Supporting our hypothesis, we saw improved behavioral performance for surface meter, as well as EEG evidence that structural meter elicits pre-attentive processing (pre-attentive P1) while surface meter does not (attentive-only P1). Our findings highlight the need for increased awareness in approaches to meter processing and support the idea that increased cognitive demand may recruit pre-attentive processing of temporal structure.
\end{abstract}

Keywords: meter processing, event-related potentials, attention, pattern recognition, pre-attentive processing 
METER WITHOUT RHYTHMIC PATTERN REPETITIONS INCREASES PRE-ATTENTIVE PROCESSING

\section{Introduction}

The temporal structure of music is based on regular events occurring at nested frequency levels and includes beat and meter. The beat level (higher frequency, shorter inter-eventintervals) comprises the isochronous time units which listeners tend to tap their foot to, and it is nested into meter. The meter level (lower frequency, longer inter-event intervals) corresponds to beat cycles, where the first beat of each cycle is strong and the others are weak (Kotz, Ravignani \& Fitch, 2018). The length of beat cycles defines different types of meter, which tend to drive dance movements. For example, a Scottish reel follows a duple meter (1-2, 1-2, ..), while a Polish mazurka is based on triple meter $(1-2-3,1-2-3, \ldots)$, triggering dancers' synchronization accordingly (e.g., Fitch, 2016). Perceiving beat and/or meter in music requires abstraction from a varying rhythmic surface (Fitch, 2013): to perceive beat, listeners must extract an isochronous pulse from multiple events that do not necessarily coincide with sound onsets; for meter, the regular recurrence of a strong beat must also be inferred from the varying rhythmic surface.

An ongoing discussion around meter processing concerns attention requirements. The dominant question has been whether meter processing is independent from attention - i.e., it can occur with or without attention, or if it requires attention. One research line focuses on meter as a source of temporal orientation in non-temporal tasks (Grahn, 2012) like, e.g., detecting pitch deviants. Results have systematically suggested that meter can be processed pre-attentively (without attention) in these circumstances (Bolger et al., 2013, 2014; Escoffier et al., 2010; Keller \& Burnham, 2005; Schwartze et al., 2011): target events presented at strong beats are detected with increased accuracy compared to events at weak beats, even when participants are unaware of meter. A different research line concerns temporal expectation - the ability to anticipate and judge the temporal onset of an event within musical time (meter as target). Here, 
METER WITHOUT RHYTHMIC PATTERN REPETITIONS INCREASES PRE-ATTENTIVE PROCESSING

results become mixed. Some studies suggest that meter is perceived under pre-attentive conditions (Bouwer et al., 2014, 2016; Geiser et al., 2010; Vuust et al., 2009; Winkler et al., 2009; Zhao et al., 2017), and direct comparisons between different levels of attention to temporal information (attention to tempo vs. pitch vs. arithmetic task) indicated similar levels of entrainment to meter (Celma-Miralles \& Toro, 2019; Lenc et al., 2020). In contrast, Geiser et al. (2009) asked participants to focus on meter itself (attentive) vs. pitch (pre-attentive), and ERPs showed that meter processing occurs only under attentive conditions. Also, even though Bower and colleagues (Bouwer et al., 2014, 2016) found evidence of pre-attentive meter processing, they found larger effect sizes for attended compared to unattended meter (Bouwer et al., 2016).

While these studies focused on testing between two alternatives - meter processing is (1) both pre-attentive and attentive vs. meter processing (2) needs attention - a recent EEG study (Silva \& Castro, 2019) targeting temporal expectation found evidence in favor of a third possibility: meter processing may be (3) pre-attentive only or, at least, it may be more effective under pre-attentive circumstances. Specifically, we found both pre-attentive and attentive structural meter processing at an early time window, and pre-attentive but not attentive processing at a later stage. This third picture emerged as meter was operationalized in a novel way, closer to the idea that meter implies abstraction (Fitch, 2013). Previous studies had used surface approaches to temporal regularities, with regular meter operationalized as the repetition of a rhythmic pattern (e.g., Geiser et al., 2009; Lenc et al., 2020; Vuust et al., 2009), and meter violations as pattern changes by adding or removing events from the original pattern (see Figure 2). In contrast, Silva and Castro (2019) adopted a structural approach to beat and meter, meaning that meter and beat violations were operationalized as deviations from underlying temporal regularities - i.e., regularities that must be abstracted from a continuously varying rhythmic 
METER WITHOUT RHYTHMIC PATTERN REPETITIONS INCREASES PRE-ATTENTIVE PROCESSING

surface (see Figures 1 and 2). In other words, the perception of meter cycles is aided by rhythmic pattern repetition in surface, but not in structural meter.

Silva \& Castro (2019) raised the possibility that the novel pattern of pre-attentive but not attentive ERPs was a response to the increased cognitive demands of structural meter (increased abstraction) compared to surface meter. Neuroimaging data (Thaut et al., 2014) have shown that different neural substrates are engaged in structural meter processing (right prefrontal and inferior frontal cortical areas) vs. pattern recognition (right temporal cortex), strengthening the notion that structural meter depends on abstraction more than surface meter. The hypothetical positive relation between cognitive demand and pre-attentive processing would be in line with the idea that using the implicit system instead of the explicit one may be a more efficient way to deal with complex sequences (Masters, 1992; Reber et al., 1980). It would also be in line with findings that meter processing for temporal orientation - i.e., while performing another task and thus under high cognitive demand - is consistently pre-attentive (Bolger et al., 2013, 2014; Escoffier et al., 2010; Keller \& Burnham, 2005; Schwartze et al., 2011), while meter processing related to temporal expectation - under less cognitive strain - may be exclusively attentive (Geiser et al., 2009).

The possibility that structural meter engages more pre-attentive processing than surface meter due to increased abstraction has not been properly tested yet, in the sense that the two types of meter have not been examined using the same paradigm and participants. To address this gap, we compared ERP responses to attended (attentive processing) vs. unattended temporal violations (pre-attentive processing) of structural vs. surface meter, using the same paradigm as Silva and Castro (2019). Attentive vs. pre-attentive responses to structural meter-related temporal violations (index of structural meter processing) were collected in one experimental 
METER WITHOUT RHYTHMIC PATTERN REPETITIONS INCREASES PRE-ATTENTIVE PROCESSING

block, and responses to surface meter-related violations (index of surface meter processing) in another block (Figure 1). A single group of participants was exposed to the two types of temporal violations, performing similar tasks in both. We predicted that the increased levels of abstraction engaged in structural meter would generate lower behavioral accuracy in discriminating between standards and deviants, as well as increased amplitude in EEG markers of pre-attentive processing (significant differences between standards and deviants under preattentive conditions).

\section{Materials and methods}

\subsection{Participants}

Twenty-eight healthy adults participated in the experiment. After excluding participants with excessive EEG artifacts, 22 were considered in the analysis ( 4 men; age: $M=22.23, S D=$ 6.91 years; schooling: $M=13.82, S D=1.53$ years). All were right-handed and did not report hearing impairments. None was taking medication or reported psychiatric or neurological disease. Nine had formal musical education $(M=6.25, S D=4.06$, range: $1-12$ years $)$. All participants signed an informed consent in line with the declaration of Helsinki.

\subsection{Stimuli}

A schematic representation of the stimulus generation process is presented in Figure 1. Each stimulus consisted of a three-bar (three beat groups) rhythmic sequence with $600 \mathrm{~ms}$ beat length and based on triple meter (3/4, see Figure 2). The duration of each sequence ranged between 5400 and $5700 \mathrm{~ms}$, depending on Stimulus type (see below). To highlight meter, the first note of each bar (strong beat) had increased intensity compared to weak beats (70 vs. $61 \mathrm{~dB}$ 
METER WITHOUT RHYTHMIC PATTERN REPETITIONS INCREASES PRE-ATTENTIVE PROCESSING

in the attack portion, see Windsor, 1993). These MIDI-generated sequences were played with a constant pitch set to G5, with a xylophone timbre (for short attacks), and they were generated using Anvil Studio (Version 2019.03.01 64 bit).

Insert Figure 1 around here, please

In each experimental block (block 1 - structural meter; block 2 - surface meter, see Figure 1), we had 29 different standards (see Appendix A) and 29 different deviants, derived from standards. Since the extraction of meter relies on detecting equal-sized groups of beats, deviant sequences were generated by changing the number of beats in the second bar. We used use Geiser et al.'s (2009) approach, where half beat (300 ms) was added to the second bar: this allowed the second bar to be perceived as a group of seven eighth-note units (7/8), contrasting with the preceding group of three quarter-note units (3/4). Participants were therefore confronted with a delayed onset for the third bar. The reason why we used delayed onsets - rather than early onsets, which have also been used in Geiser et al. (2009) and Silva and Castro (2019) - was that Silva and Castro (2019) showed a correlation between behavior and EEG for delayed, but not for early onsets.

Structural meter standards (Figures 1-2i) had regular meter (three 3/4 bars, meaning three beats per each of the three cycles) and regular beat length (all beats $=600 \mathrm{~ms}$ ). The first and third (last) bars were composed of eighth notes that marked the beat onset, while the second bar was composed of varying rhythmic phrases using quarter-, eighth-, and sixteenth notes (see Appendix A). In structural meter deviants (Figures 1-2ii), the second bar was transformed into a longer, 7/8 bar. Changing the rhythmic pattern from the first to the second bar in both standard 
METER WITHOUT RHYTHMIC PATTERN REPETITIONS INCREASES PRE-ATTENTIVE PROCESSING

and structural meter deviant versions was critical to our design, in that it granted that listeners would not rely on pattern changes to detect deviants.

Surface meter standards (Figures 1-2iii) were generated by copying the rhythm of the second bar into the first and third bars, meaning that rhythmic phrases were the same for all three bars. Similar to structural meter deviants, surface meter deviant versions were generated by adding an eighth note at the end of the second bar of surface meter standards, therefore increasing its length by $300 \mathrm{~ms}$ and transforming the second bar into 7/8 meter (Figures 1-2iv). In contrast to structural meter deviants, these surface meter deviants could be detected based on pattern changes (the same pattern with one event added).

The two experimental blocks (structural meter standards vs. deviants; surface meter standards vs. deviants) were presented twice: first in a pitch-deviant detection task (pre-attentive temporal processing condition) and then in an explicit temporal violation detection task (attentive temporal processing condition). To make the pitch-detection task possible, we lowered the pitch of one note to $\mathrm{C} 5$ in $33 \%$ of the sequences (both standards and deviants). The target note was always the second beat of the third bar to guarantee that meter and beat deviant detection (occurring before, at the second bar) was not disturbed.

Insert Figure 2 around here, please

Each block had a set of triggers (Tg) that marked its critical events (Figure 2). In the first block (structural meter standard vs. deviants), a trigger was placed at the onset of the third bar of standard stimuli, capturing the third bar onset (Tg1), and another was placed $300 \mathrm{~ms}$ later for meter-deviant stimuli, capturing the delayed third bar onset $(\operatorname{Tg} 2)$. In the second block 
METER WITHOUT RHYTHMIC PATTERN REPETITIONS INCREASES PRE-ATTENTIVE PROCESSING

(surface meter standards vs. deviants), triggers were placed similarly to the structural conditions

( Tg3 and Tg4). Therefore, in both blocks, we intended to capture the correlates of delayed onsets relative to listeners' expectations and the corresponding perception of irregularity in the preceding temporal unit.

\subsection{Procedure}

In both structural meter and surface meter blocks, participants were presented the same set of temporal standards vs. deviants twice, once for each of the two tasks. In the pitch-deviant detection task, participants were asked to detect sequences with a pitch alteration, and therefore listened to temporal violations pre-attentively. In the temporal-violation detection task, participants were asked to detect temporal deviants (beat or meter-related, depending on the block), thus processing temporal structure in an attentive manner.

To minimize attention to meter in the pre-attentive conditions, all participants started with the pitch-deviant detection task, where they were instructed to press a key if they detected a lower-pitched note in the stimulus, and another key if they did not. Afterwards, participants completed the temporal-violation detection tasks, in which participants were asked to detect if there was a longer bar. Before these tasks, they were given brief verbal and graphic explanations on the concepts of beat, meter and bar, and they were presented with examples of standard and deviant sequences. Each block included three practice trials, which could be repeated until participants confirmed that they understood the task.

Each trial consisted of the following sequence: a 200 ms fixation cross, an auditory stimulus (between 5400 and $5700 \mathrm{~ms}$, depending on the condition), a written question on the screen ("Is there a low-pitched note, different from all the others?", or "Did the meter change? "). 
METER WITHOUT RHYTHMIC PATTERN REPETITIONS INCREASES PRE-ATTENTIVE PROCESSING

Each question called for a keypress, to which participants answered 'yes' or 'no' in a self-paced manner. The next trial started immediately after the keypress. The assignment of keys to responses was balanced across participants. Participants had a break between each of the four tasks (two tasks per block).

Block order was balanced across participants. Stimuli were pseudorandomized four times (one pseudo randomization per block). For each pseudo randomization, two stimulus orderings were created (A and B), with B having the order of halves switched compared to A (A, 1-29; B, 15-29 followed by 1-14). Half of the participants listened to versions A, and the other half to versions B. Stimuli were delivered through loudspeakers in an acoustically shielded room.

Before starting the experiment, participants signed the informed consent forms and filled in a sociodemographic questionnaire. The total duration of the experiment ranged between 50 and 70 minutes.

\subsection{EEG recording and preprocessing}

Participants sat comfortably in front of the stimulation computer. They were asked to move as little as possible during the tasks and to blink between trials. EEG data were collected using a Biosemi ActiveTwo system at a $512 \mathrm{~Hz}$ digitization rate. Sixty-four active channels were mounted on a BioSemi elastic headcap following the 10-20 system. Electrodes were referenced to two external electrodes placed at the right and left mastoid bones, and the Biosemi default ground electrodes (CMS and DRL) were used. Another external electrode was placed under the left eye to detect vertical EOG artifacts. During electrode placement, signal quality was kept under the system-recommended thresholds. 
METER WITHOUT RHYTHMIC PATTERN REPETITIONS INCREASES PRE-ATTENTIVE PROCESSING

EEG data were preprocessed using the Fieldtrip toolbox (Oostenveld et al., 2011) for MATLAB. Epochs were extracted by marking $200 \mathrm{~ms}$ before and $1000 \mathrm{~ms}$ after trigger points (see Stimulus section). Epoch-based artefact rejection was then performed in two stages: first, EOG artefacts were removed through inspection of variance in vertical (EOG external electrode bipolarly referenced to Fp1) and horizontal movements (F7 referenced to F8); second, trial variance was inspected to remove epochs containing other artefacts, and also to remove defective channels. Epochs were then baseline corrected (baseline $=200 \mathrm{~ms}$ ), detrended, re-referenced to the mastoid electrodes, and band-pass filtered between 0.01 and $30 \mathrm{~Hz}$. Removed channels were interpolated using nearest neighborhood averaging. Epochs were averaged for each condition per subject, and these were grand averaged.

\subsection{Statistical analysis}

We examined behavioral results using d-prime, an index of discrimination (Stanislaw \& Todorov, 1999). We compared participants' performance across the two temporal conditions (structural meter and surface meter) in each of the two tasks (pitch vs. meter deviant detection) using separate ANOVAs. To determine potential influences from musical training, which have been reported for meter processing (Geiser et al. 2009, Geiser et al., 2010; Palmer \& Krumhansl, 1990; Perna et al., 2018; Yates et al., 2016 for meter, but see Bower et al., 2014), we added musical training (yes vs. no) as a between-subjects factor (mixed ANOVAs). In addition, we checked whether the behavioral performance of participants without musical training was above chance levels, using one-sample tests against zero.

Concerning EEG data, we started by grouping grand averaged channels into 9 Regions of Interest (ROIs), following Silva and Castro (2019): right anterior (Fp2, AF8, AF4, F4, F6), 
METER WITHOUT RHYTHMIC PATTERN REPETITIONS INCREASES PRE-ATTENTIVE PROCESSING

middle anterior (F1, Fpz, AFz, Fz, F2), left anterior (FP1, AF7, AF3, F3, F5), right central (FC6, FC4, C4, C6, CP6, CP4), middle central (FC1, C1, CP1, CPz, Cz, FCz, FC2, C2, CP2), left central (FC5, FC3, C3, C5, CP5, CP3), right posterior (P4, P6, P8, PO8, PO4), middle posterior (P1, Oz, POz, Pz, P2), and left posterior (P3, P5, P7, PO7, PO3).

Visual inspection of the waveforms highlighted increased positivity for deviants along the entire epoch for structural meter and surface meter processing. This ruled out the presence of a negative component marking deviance perception, which has been reported in some studies (MMN, see Geiser et al., 2009, or N2b, Bower et al., 2016). Therefore, we focused on the series of visible positive peaks matching commonly reported auditory ERPs (Winkler et al., 2013), which we referred to as P1 (80-120 ms), P2 (150-275 ms), P3 (300-500 ms) and P600 ms (600$800 \mathrm{~ms}$, see (Friederici, 2002). Along with MMN or N2b, the literature on meter processing often refers to an increased P3 component in deviants (Bower et al., 2016), though its latencies (180-260 ms) resemble those of our P2. For each block and time window, we ran 2 × 2 × 3 × 3 within-subjects ANOVAs, comprising Attention (attended vs. unattended), Stimulus type (standard vs. deviant), Caudality (anterior, central, posterior), and Laterality (left, middle, right) as factors. To allow a direct comparison with Silva \& Castro's (2019) study, we made a complementary analysis based on $300 \mathrm{~ms}$ time windows (TWs): 0-300 ms, 300-600 ms, 600-900 ms.

Since our goal was to investigate the balance between pre-attentive and attentive processing in structural meter vs. surface meter, we focused on attention x stimulus type interactions and further interactions with topographic factors (attention x stimulus type $\mathrm{x}$ caudality, laterality, or caudality x laterality) for both types of temporal structure (structural meter and surface meter). The highest-order interaction was broken down to locate the regions 
METER WITHOUT RHYTHMIC PATTERN REPETITIONS INCREASES PRE-ATTENTIVE PROCESSING

where attention $\mathrm{x}$ stimulus type interactions occurred: for instance, when both attention $\mathrm{x}$ stimulus type and attention $\mathrm{x}$ stimulus type $\mathrm{x}$ caudality were significant, we broke down the latter. Once located, these regions were analyzed for stimulus type effects per attention condition. We also examined the main effects of stimulus type, which would be particularly important in the absence of attention x stimulus type interactions. In this case, significant main effects of stimulus type or interactions of these with topographic factors would indicate attention-independent (attentive and pre-attentive) temporal processing, while non-significant effects would point to the absence of EEG signatures of temporal processing, whether attentive or pre-attentive. After separate analyses of each temporal structure, we made direct comparisons between temporal structures to strengthen the validity of the hypothetical differences in attention requirements.

In a complementary test of the hypothesis that increased cognitive demand goes along with increased presence of pre-attentive processing, we compared high vs. low d-prime participants (defined by median split) for their use of attentive vs. pre-attentive processing (attention $\mathrm{x}$ stimulus type interaction in each group). We predicted that, whenever pre-attentive processing was present, low d-prime participants (those with more difficulty in performing the task) would show increased stimulus type effects for the unattended condition compared to attended, while high d-prime participants would show the reverse pattern.

All statistical decisions were guided by conventional null-hypothesis-significance-testing, based on the traditional threshold for significance of alpha $<.05$ with sphericity violations handled with Greenhouse-Geiser corrections. We also calculated Bayes Factors (BF) in favor of the alternative hypothesis over the null one $\left(\mathrm{BF}_{10}\right)$, with JASP software (JASP team, 2020) and using default priors. Unlike traditional null-hypothesis-significance-testing, which relies on 
METER WITHOUT RHYTHMIC PATTERN REPETITIONS INCREASES PRE-ATTENTIVE PROCESSING

dichotomous information (significant vs. non-significant results), Bayes factors quantify the relative predictive performance of two alternative hypothesis (alternative vs. null, or null vs. alternative), measuring the strength of evidence in favor of one over the other (Biel \& Friedrich, 2018; van Doorn et al., 2020). Bayes factors are particularly relevant to strengthen claims of null effects and clarify marginal results, and this was how we have mostly used them in the present study. Following the heuristics provided in van Doorn et al. (2020), we considered BFs between 1 and 3, 3 and 10, 10 and 30 and above 30 as weak, moderate, strong and very strong evidence in favor of the alternative hypothesis. While BFs above 1 support the alternative hypothesis, BFs below 1 indicate evidence in favor of the null hypothesis, and evidence here becomes stronger as values decrease: BFs between 1 and 0.33 provided weak evidence, between 0.33 and 0.10 moderate, between 0.10 and 0.03 strong, and below 0.03 very strong.

\section{Results}

\subsection{Behavioral results}

In the pitch deviant detection task (pre-attentive temporal processing), Wilcoxon signedrank tests against zero showed above-chance discrimination for both structural meter $(M=7.16$, $S D=0.73, p<.001)$ and surface meter $(M=4.45, S D=1.99, p<.001)$, with very strong $B F_{10 s}$ (> 30). Notwithstanding, the mixed ANOVA with meter type and musical training as factors (Figure 3) indicated improved pitch detection for structural meter, $F(1,20)=105.13, p=.004, \eta^{2} p$ $=0.031, B F_{10}>30$. Musical training had no significant effects, $F(1,20)=0.12, p=.737, \eta^{2}{ }^{2}=$ $0.006, B F_{10}=0.393$, and it did not interact with meter type, $F(1,20)=0.64, p=.432, \eta^{2}{ }^{2}=0.031$, $B F_{10}=0.560$. 
METER WITHOUT RHYTHMIC PATTERN REPETITIONS INCREASES PRE-ATTENTIVE PROCESSING

In the meter deviant detection task (attentive temporal processing), one-sample tests against zero also showed above-chance temporal discrimination for both meter types (structural meter: $M=2.05, S D=1.54, t(21)=6.24, p<.001$; surface meter: $M=2.87, S D=1.95, \mathrm{~V}=231$, $p<.001)$. Bayesian evidence was very strong in both cases $\left(B F_{10}>30\right)$. The mixed ANOVA (Figure 3) showed a main effect of meter type, $F(1,20)=6.98, p<.016, \eta^{2} p=0.259, B F_{10}=$ 2.681 and no significant interaction with musical training, $F(1,20)=0.49, p=.493, \eta^{2}{ }_{p}=0.024$, $B F_{10}=0.780$ : d-prime values for structural meter were overall lower than for surface meter. Effects of musical training were non-significant, $F(1,20)=4.06, p=.057, \eta^{2} p=0.169$, but the Bayes factor pointed to weak evidence in favor of the alternative hypothesis $\left(B F_{10}=1.474\right)$. Therefore, we ran one-sample tests against zero for participants without musical training. We found above-chance performance for both surface, $t(13)=4.47, p<.001, B F_{10}>30$, and structural meter, $\mathrm{V}=91, p<.001, B F_{10}>30$, indicating that the absence of musical training does not prevent successful meter discrimination. This prompted us to not consider musical training as a relevant factor in EEG analysis.

In sum, participants performed better in the pitch deviant detection task when they were listening to structural meter sequences compared to when they were listening to surface meter ones. Concerning differences in meter deviance detection across meter types, increased performance for surface meter compared to structural meter supports our first hypothesis. It is worth noting that d-prime values for structural meter reached much higher values here, $M=2.05$, than in Silva and Castro (2019), $M=0.49$. Why would the same task, performed by a sample of participants with similar characteristics (five out of eighteen participants with musical training, ranging from 1 to 6 years of training), induce such differences in performance? This may be 
METER WITHOUT RHYTHMIC PATTERN REPETITIONS INCREASES PRE-ATTENTIVE PROCESSING

evidence in favor of our hypothesis of increased pre-attentive processing under increased cognitive demand: Silva and Castro (2019) presented three different types of meter deviance (delayed onset, early onset and silent onset) instead of one (delayed onset, as here), and this may have made the task more difficult than in the current study.

\subsection{Structural meter processing: pre-attentive and attentive at early stages}

Time Window 1 (80-120 ms, P1, Figure 4): the attention $\mathrm{x}$ stimulus type interaction was non-significant, $F(1,21)=0.02, p=.910, \eta^{2}{ }_{p}<.001, B F_{10}=0.043$, and so were further interactions with laterality or caudality $\mathrm{x}$ laterality, $p \mathrm{~s}>.135, \eta^{2}{ }_{p s}<0.097, B F_{s 10}<0.152$. A significant attention $\mathrm{x}$ stimulus type $\mathrm{x}$ caudality interaction was apparent, $F(1.14,23.98)=10.88$, $p=.002, \eta_{p}^{2}=0.341, B F_{10}=0.152$, but all caudality levels showed non-significant attention $\mathrm{x}$ stimulus type or attention $\mathrm{x}$ stimulus type $\mathrm{x}$ laterality interactions (anterior: $p s>.158, \eta_{p s}^{2}<$ 0.009, BFs $10<7.240$; central: $p s>.096, \eta_{p s}^{2}<0.119, B F s_{10}<0.060$; posterior: $p s>.125, \eta_{p s}^{2}<$ 0.107, $B F s_{10}<3.410$ ). When searching for main effects of stimulus type (attention-independent meter processing), we found a significant effect, $F(1,21)=4.89, p=.038, \eta^{2}{ }_{p}=0.189$, associated with a very strong Bayes factor $\left(B F_{10}>100\right)$, and non-significant interactions with topographic factors, $p s>.264, \eta_{p s}^{2}<0.003, B F s_{10}<0.024$. This effect is compatible with an increased P1 component for beat violations (standard, $\mu V, M=-0.88, S E=0.79,95 \% C I=[-2.28,0.72]$; deviant, $M=0.90, S E=0.48,95 \% C I=[0.05,1.81])$.

Overall, the analysis suggests that attention-independent processing is occurring all over the scalp in this early time window. Note that, although Bayesian evidence partly contradicted the null interactions engaging attention and stimulus type shown by frequentist analyses $\left(B F s_{10}\right.$ 
METER WITHOUT RHYTHMIC PATTERN REPETITIONS INCREASES PRE-ATTENTIVE PROCESSING

reaching 7.240 and 3.410 at anterior and posterior regions), this evidence was comparatively much stronger when analyzing attention-independent processing $\left(B F_{10}>100\right)$.

Time Window 2 (150-275 ms, P2): the attention x stimulus type interaction was nonsignificant, $F(1,21)=.09, p=.76, \eta^{2}{ }_{p}=.004, B F_{10}=0.160$, but interactions with caudality, $F(1.08,22.58)=5.95, p=.020, \eta^{2}{ }_{p}=0.221, B F_{10}=0.275$, and laterality, $F(1.41,29.56)=3.93, p$ $=.044, \eta_{p}^{2}=0.158, B F_{10}<0.001$, were apparent. When we inspected attention $\mathrm{x}$ stimulus type effects in each caudality level, all results were null, $p \mathrm{~s}>.21, \eta_{p s}^{2}<0.073, B F_{s 10}<1.95$, but an interaction with laterality was significant for central electrodes, $F(1.32,27.63)=6.21, p=.013$, $\eta_{p}^{2}=.228, B F_{10}<0.001$. Likewise, when searching for attention $\mathrm{x}$ stimulus type effects across laterality levels, none was apparent, $p \mathrm{~s}>.55, \eta^{2}{ }_{p s}<0.017, B F_{s 10}<0.102$, but a second order interaction with caudality emerged for right, $F(1.18,24.72)=7.09, p=.01, \eta^{2}{ }_{p}=0.252, B F_{10}=$ 0.009 , and middle electrodes, $F(1.11,23.21)=6.82, p=.01, \eta^{2}{ }_{p}=0.245, B F_{10}=0.008$. We broke down the analysis into specific regions, searching for attention x stimulus type effects at right central $(\mathrm{RC})$ and middle Central $(\mathrm{MC})$ electrodes, but none reached significance $(\mathrm{RC}: F(1,21)=$ $0.339, p=.567, \eta_{p}^{2}=0.016, B F_{10}=0.226 ; \mathrm{MC}: F(1,21)=0.181, p=.67, \eta^{2} p=0.009, B F_{10}=$ 0.167). Therefore, no attention-dependent effect emerged in this time window. Regarding attention-independent effects, we saw a significant stimulus type $\mathrm{x}$ caudality $\mathrm{x}$ laterality interaction, $F(2.57,53.88)=3.73, p=.021, \eta^{2}=0.151, B F_{10}<0.001$. When breaking the analysis into individual regions, attention-independent effects emerged for right central, $F(1,21)$ $=5.23, p=.033, \eta_{p}^{2}=.199, B F_{10}=2.09$, right posterior, $F(1,21)=5.98, p=.023, \eta_{p}^{2}=.222$, $B F_{10}=0.234$, and middle posterior regions, $F(1,21)=4.85, p=.04, \eta^{2}=0.188, B F_{10}=1.890$. In sum, an attention-independent P2 effect was apparent at right central, right posterior, and middle posterior electrodes, associated with increased positivity for meter violations (e.g., 
METER WITHOUT RHYTHMIC PATTERN REPETITIONS INCREASES PRE-ATTENTIVE PROCESSING

right central: standard, $\mu V, M=-1.74, S E=0.77,95 \% C I=[-3.29,-0.26]$; deviant, $M=0.50$,

$S E=0.76,95 \% C I=[-1.02,1.85])$. As we saw above, Bayesian evidence contradicted the narrow topography pointed out by frequentist analyses, suggesting a widespread increased P2 for deviants.

Time Window 3 (300-500 ms, P3): the attention x stimulus type interaction was nonsignificant, $F(1,21)=0.174, p=.68, \eta^{2}=0.008, B F_{10}=0.029$, and so were further interactions with topographic factors, $p \mathrm{~s}>.11, \eta_{p s}^{2}<0.112, B F_{s 10}<0.001$. There were no significant stimulus type effects, $F(1,21)=0.119, p=.73, \eta^{2}{ }_{p}=0.006, B F_{10}=0.022$ or further interactions with topographic factors, $p \mathrm{~s}>.58, \eta_{p s}^{2}<0.028, B F_{s 10}<0.004$.

In short, no relevant effects emerged in this time window, hinting that P3 may not be an appropriate component for indexing structural meter processing.

Time Window 4 (600-800 ms, P600): the attention $\mathrm{x}$ stimulus type interaction failed to reach significance, $F(1,21)=1.37, p=.26, \eta_{p}^{2}=0.061, B F_{10}=15.230$, and so did interactions with laterality and with caudality x laterality, $p \mathrm{~s}>.16, \eta_{p s}^{2}<0.082, B F_{s 10}<0.001$, but a significant attention $\mathrm{x}$ stimulus type $\mathrm{x}$ caudality emerged $\left(F(1.21,25.31)=5.60, p=.02, \eta^{2}{ }^{2}=\right.$ $0.210, B F_{10}=7.62$. When breaking the analysis into the three caudality levels, no significant attention $\mathrm{x}$ stimulus type emerged for anterior electrodes, $F(1,21)=.025, p=.88, \eta^{2} p=.001$, $B F_{10}=0.008$, and only marginal effects were visible for central ones, $F(1,21)=3.62, p=.08$, $\eta_{p}^{2}=0.147, B F_{10}=22.44$. In contrast, posterior electrodes showed a significant attentiondependent effect, $F(1,21)=7.09, p=.02, \eta^{2}{ }_{p}=0.252, B F_{10}>100$. Breaking the analysis into attention levels, increased positivity for deviants showed up in the attended condition, $F(1,21)=$ 9.76, $p=.005, \eta_{p}^{2}=0.317, B F_{10}>100$ (standard, $\mu V, M=-0.22, S E=0.43,95 \% C I=[-1.01$, 0.64]; deviant, $M=1.39, S E=0.44,95 \% C I=[0.60,2.31])$, but not in the unattended 
METER WITHOUT RHYTHMIC PATTERN REPETITIONS INCREASES PRE-ATTENTIVE PROCESSING

condition, $F(1,21)=.017, p=.90, \eta_{p}^{2}<0.001, B F_{10}<0.001($ standard, $\mu V, M=-0.75, S E=$ $0.31,95 \% C I=[-1.36,-0.14 ;$ deviant $, M=-0.81, S E=0.38,95 \% C I=[-1.52,-0.12])$. This attentive-only increased positivity for structural meter violations is compatible with a more expressive P600 component. One should note, however, that there was strong Bayesian evidence that this increased positivity for meter violations may not exclusively arise from posterior electrodes, given the strong Bayes Factor associated with the whole scalp analysis $\left(B F_{10}=\right.$ 15.23), and with the central region $\left(B F_{10}=22.44\right)$.

In sum, meter processing was attentive-only in the P600 time window.

Insert Figure 4 around here, please

Overview: structural meter processing was attention-independent at an early stage, as supported by increased P1 and P2 components under both attended and unattended conditions. At a later stage, structural meter showed a more pronounced P600 for deviants in attended, but not unattended conditions. Thus, pre-attentive processing was present at earlier time windows, but absent between 600 and 800 ms. Between 300 and 500 ms, neither attentive nor pre-attentive processing signatures were observed. Analyses of correct trials only (Appendix B) showed the same pattern of results. Complementary analyses based on $300 \mathrm{~ms}$ time windows for comparison with Silva and Castro (2019) showed marginal attention-independent effects for 0 -300 and 300$600 \mathrm{~ms}$ (without attention x stimulus type interactions), and significant attention-dependent effects (attentive only) between 600 and 900 ms (Appendix C). Note that Silva and Castro's (2019) analysis was restricted to the 0-300 and 300-600 ms time windows, with results showing 
METER WITHOUT RHYTHMIC PATTERN REPETITIONS INCREASES PRE-ATTENTIVE PROCESSING

attention-independent processing between 0 and $300 \mathrm{~ms}$ and pre-attentive-only processing between 300 and $600 \mathrm{~ms}$.

\subsection{Surface meter processing: attentive-only in all time windows}

Time Window 1 (80-120 ms, P1, Figure 5): a significant attention x stimulus type interaction, $F(1,21)=21.71, p<.001, \eta^{2}{ }_{p}=0.508, B F_{10}>100$, was observed (Figure 5). In the attended condition, there was a significant stimulus type effect across the whole scalp (increased positivity for deviants: $F(1,21)=6.37, p=.02, \eta_{p}^{2}=0.233, B F_{10}>100$; standard, $M=-1.02$, $S E=0.57,95 \% C I=[-2.24,0.13] ;$ deviant $, M=1.80, S E=0.86,95 \% C I=[0.25,3.47])$. In contrast, the unattended condition showed non-significant stimulus type effects, $F(1,21)=.06, p$ $=.81, \eta_{p}^{2}=0.003, B F_{10}=006$ (standard, $M=0.58, S E=0.79,95 \% C I=[-0.81,2.20] ;$ deviant , $M=0.37, S E=0.49,95 \% C I=[-0.53,1.45])$.

Time Window 2 (150-275 ms, P2): results were similar to those for TW 1. A significant attention $\mathrm{x}$ stimulus type interaction, $F(1,21)=21.71, p<.001, \eta^{2}{ }_{p}=0.508, B F_{10}>100$, was observed, indicating significant stimulus type effects (increased positivity for deviants) across the whole scalp for attended, $F(1,21)=8.72, p=.008, \eta^{2} p=0.293, B F_{10}>100($ standard, $M=-$ 1.06, $S E=0.64,95 \% C I=[-2.32,0.19] ;$ deviant, $M=2.52, S E=1.11,95 \% C I=[0.35,4.64])$, but not for unattended surface meter, $F(1,21)=.37, p=.55, \eta_{p}^{2}=0.017 B F_{10}=1.580$ (standard, $M=-0.54, S E=0.92,95 \% C I=[-0.22,1.18] ;$ deviant $, M=-1.15, S E=0.45,95 \% C I$ $=[-2.00,-0.26])$.

Time Window 3 (300-500 ms, P3): the attention x stimulus type interaction was significant, $F(1,21)=5.27, p=.03, \eta^{2} p=0.201, B F_{10}>100$. The unattended condition showed clearly non-significant stimulus type effects, $F(1,21)=0.42, p=.53, \eta^{2} p=0.019, B F_{10}<0.010$ (standard, $M=-1.16, S E=0.83,95 \% C I=[-2.73,0.47] ;$ deviant, $M=-1.75, S E=0.59,95 \% C I$ 
METER WITHOUT RHYTHMIC PATTERN REPETITIONS INCREASES PRE-ATTENTIVE PROCESSING

$=[-2.77,-0.55])$, but - unlike previous time windows - effects for the attended condition were only marginal (increased positivity for violations, $F(1,21)=3.70, p=.060, \eta^{2}{ }_{p}=0.149 ; B F_{10}>$ 100 (standard, $M=-1.15, S E=0.99,95 \% C I=[-2.98,0.94] ;$ deviant $, M=1.77, S E=1.11,95 \%$ $C I=[-0.35,4.00])$. Since marginal effects went along with a very strong Bayes factor $(>100)$, we endorsed stimulus type effects in the attended condition.

TW 4 (600-800 ms, P600): again, we found a significant attention x stimulus type interaction, $F(1,21)=13.13, p=.002, \eta^{2} p=0.39, B F_{10}>100$. In the attended condition, there was a significant stimulus type effect (increased positivity for deviants) across the whole scalp, $F(1,21)=21.75, p<.001, \eta_{p}^{2}=0.001, B F_{10}>100($ standard $, M=-1.14, S E=0.42,95 \% C I=$ $[-2.00,-0.35]$; deviant, $M=1.57, S E=0.67,95 \% C I=[0.18,2.69])$. In the unattended condition, stimulus type effects were non-significant, $F(1,21)=.06, p=.81, \eta^{2} p=0.003, B F_{10}=$ 0.06 (standard, $M=0.07, S E=0.64,95 \% C I=[-1.29,1.30] ;$ deviant, $M=-0.9, S E=0.38,95 \%$ $C I=[-0.80,0.69])$.

Insert Figure 5 around here, please

Overview: attention effects on surface meter processing were present in all time windows, reflected as a widespread increased positivity for surface meter violations under the attended condition, but not under the unattended condition. This result suggests that pre-attentive processing is not recruited for surface meter. Analyses of correct trials only (Appendix B) showed the same pattern of results. Complementary analyses based on $300 \mathrm{~ms}$ segments (Appendix D) also showed the same pattern of results, with attention-dependent processing in all time windows. 
METER WITHOUT RHYTHMIC PATTERN REPETITIONS INCREASES PRE-ATTENTIVE PROCESSING

\subsection{Direct comparisons between surface and structural meter}

Direct comparisons between structural and surface meter were made for the time windows where both types of temporal structure showed significant meter processing (P1, P2 and P600), and they were based on the smallest region showing stimulus type effects. The 300-500ms time window (P3) was not considered in this analysis, given that no attention-related processing signature was significant for structural meter. Differences in attention requirements were expected for P1 and P2 (pre-attentive processing in structural, but not surface meter). No differences were expected for P600, since both meter types showed attentive processing only.

The P1 time window (analyzed at whole-scalp level) showed a significant temporal structure $\mathrm{x}$ attention $\mathrm{x}$ stimulus type $\mathrm{x}$ caudality interaction, $F(1.07,22.66)=6.57, p=.016, \eta^{2} p=0.238$. When breaking the analysis into caudality levels, we saw significant temporal structure $\mathrm{x}$ attention $\mathrm{x}$ stimulus type interactions in anterior, $F(1.07,22.66)=4.33, p=.039, \eta_{p}^{2}=0.187$, though not in central or posterior electrodes (central: $F(1,21)=3.43, p=.078, \eta^{2} p=0.140$; posterior: $\left.F(1,21)=0.76, p=.785, \eta^{2} p=0.004\right)$. Bayes factors could not be computed due to unavailable computational power to process the very large number of models generated. The analysis validates the idea that structural meter recruits pre-attentive processing, while surface meter does not. The same did not happen in the P2 time (analyzed for right middle, right posterior and middle posterior regions), since interactions containing temporal structure $\mathrm{x}$ attention $\mathrm{x}$ stimulus type failed to reach significance, $p_{s}>.126, \eta_{p s}^{2}<0.104, B F_{10}<0.254$. Finally, the P600 time window, analyzed for posterior channels, showed the expected nonsignificant result concerning the temporal structure $\mathrm{x}$ attention $\mathrm{x}$ stimulus type interaction, 
METER WITHOUT RHYTHMIC PATTERN REPETITIONS INCREASES PRE-ATTENTIVE PROCESSING

$F(1,21)=0.41, p=.531, \eta_{p}^{2}=0.019, B F_{10}=0.644$. This indicates equivalent attentive-only

processing in surface and structural meter, thus validating the results from individual analyses.

In sum, comparative results for the P1 but not the P2 time window validated the idea that structural, but not surface meter, requires increased pre-attentive processing.

\subsection{Attention effects according to behavioral performance}

To cross-test the hypothesis that increased cognitive demand goes along with increased preattentive processing, we added d-prime (high vs. low) to the analysis of time windows where pre-attentive processing was observed. This included the P1 and P2 time windows in structural meter processing (Figure 6).

For the P1 time window (attentive and pre-attentive processing at whole scalp), we found a significant attention $\mathrm{x}$ stimulus type $\mathrm{x}$ d-prime interaction, $F(1,21)=6.77, p=0.017, \eta^{2}{ }_{p}=0.253$, indicating reliance on pre-attentive processing in low d-prime participants and reliance on attentive processing in the high d-prime group. Note that the stimulus type $\mathrm{x}$ d-prime interaction was not significant, $F(1,21)=1.33, p=.262, \eta^{2} p=0.062$, suggesting that EEG indices of meter discrimination did not differ across groups: instead, they differed in the balance between attentional and pre-attentional processing used to achieve discrimination.

For the P2 time window (attentive and pre-attentive processing at right central, right posterior and middle posterior regions, the attention $\mathrm{x}$ stimulus type $\mathrm{x}$ d-prime interaction did not reach significance, $F(1,21)=1.44, p=0.243, \eta^{2}=0.067$, although the balance between attentive and pre-attentive processing for low vs. high d-prime participants seemed to follow a pattern similar to that found for P1. Again, low and high d-prime participants did not differ in EEG indices of discrimination (stimulus type $\mathrm{x}$ d-prime interaction: $F(1,21)=2.24, p=0.150, \eta^{2}{ }_{p}=$ 
METER WITHOUT RHYTHMIC PATTERN REPETITIONS INCREASES PRE-ATTENTIVE PROCESSING

0.101). Again, we were not able to compute Bayes factors due to insufficient computational power to handle the large number of models induced by adding a between-subjects factor.

Insert Figure 6 around here, please

In sum, cross-tests comparing low and high d-prime participants also strengthened the idea that structural meter requires more pre-attentive processing than surface meter. Similar to the direct comparisons reported above, the P1 but not the P2 time window carried the effect.

\section{Discussion}

Ongoing discussions regarding the influences of attention on meter processing have remained focused on one of two alternatives: either meter requires attention (it can only be processed attentively) or it does not, meaning that meter can be processed both attentively and preattentively. This approach is often framed by a debate on whether meter is fundamental to human cognition: attention requirements would speak against meter as a fundamental ability (see, e.g., Winkler et al., 2009). The hypothesis of attentive-only meter processing is also linked to theories of timing, according to which longer (suprasecond) time units may require more cognitive control, and hence more attention than shorter units such as beats (Grahn, 2012). From these viewpoints, increased complexity would require more attention.

In the current paper, we adopted the opposite perspective: could it be that the cognitive load inherent to some forms of meter processing requires lack of attention, i.e., that pre-attentive processing of meter is a response to increased cognitive demand? We raised this possibility based on two sets of findings. First, meter processing in a dual-task context (meter for temporal orientation) is consistently pre-attentive, while meter processing per se (meter as temporal 
METER WITHOUT RHYTHMIC PATTERN REPETITIONS INCREASES PRE-ATTENTIVE PROCESSING

expectation) has shown benefits of attention. Second, processing complex forms of meter (structural meter, not aided by pattern repetition) requires pre-attentive processing (Silva \& Castro, 2019), while simpler forms of meter (surface meter, aided by pattern repetition) are processed attentively.

We found converging evidence in favor of our hypothesis. First, behavioral results showed that surface meter is easier to process then structural meter, thus strengthening the idea that surface meter requires less abstraction. Second, EEG results showed that pre-attentive processing is not engaged in surface meter processing, but it exists in the earlier stages of structural meter processing. Third, we found partial evidence that, when both attentive and pre-attentive processing are present, participants with lower behavioral discrimination (increased difficulty in dealing with meter processing) rely mostly on pre-attentive processing, while high-performing individuals do the opposite. Four and final, comparisons between the present EEG results and those of Silva and Castro (2019) highlight the fact that Silva and Castro (2019) obtained lower behavioral performances (d-prime $=0.49$, against 2.05 in the current study) and this lower performance co-occurred with increased reliance on pre-attentive processing (pre-attentive only, while we had both attentive and pre-attentive in similar time windows). Altogether, these findings converge on the idea that cognitive demand goes along with reliance on pre-attentive processing of meter.

Despite the observed differences in attention patterns for structural vs. surface meter, the ERP components that marked the processing of both temporal structures were rather similar: in both, we found increased positivity for deviants between 80 and $120 \mathrm{~ms}$ (a first positive peak, P1) and between 150 and 275 ms (second positive peak, P2). These peaks were attentionindependent for structural meter and attentive only for surface meter. Increased P1 and P2 
METER WITHOUT RHYTHMIC PATTERN REPETITIONS INCREASES PRE-ATTENTIVE PROCESSING

components have been reported in response to temporal irregularities (Neuhaus \& Knösche, 2006), consistent with our findings. Also in both structural and surface meter, we found a late positive peak between 600 and 800 ms, larger for deviants and resembling the P600 (Hagoort \& Brown, 2000; Friederici, 2002). This component was attentive-only in both cases. The P600 has been related to syntactic-like violations - not only in speech, but also in music (e.g., irregular chord sequences, see Friederici et al., 2002) - namely as an index of revision and integration following deviance detection. It is possible that this component reflects participants' revision and integration of the deviant meter cycle (the $7 / 8$ meter cycle) in the $3 / 4$ cycle context. Another possibility is that the positive peak reflected the onset of the next beat (second beat of third bar). In the 300-500 ms time window, a third positive peak - again increased for deviants - was apparent in structural as well as surface meter processing (P3), but it was significant only for surface meter (attentive-only processing). Among these components, P1 - more than P2 seemed to have carried the difference between structural and surface meter processing: for P1, but not for P2, we saw significant differences when doing direct comparisons. For P1, but not for P2, we found different patterns of attention in low vs. high d-prime participants. As for P600, its relation with attentive processing survived direct comparisons.

The results we obtained for structural meter are in line with Silva \& Castro's (2019) study, where pre-attentive processing was observed between 0 and $600 \mathrm{~ms}$. One difference regards the fact that Silva and Castro (2019) found pre-attentive-only processing between 300 and $600 \mathrm{~ms}$, while here processing was both attentive and pre-attentive. As pointed out above, this would be consistent with the fact that Silva and Castro engaged participants in detecting three different types of meter deviance detection and, thus, the task was more demanding than the current one. Behavioral results confirmed this, in that d-prime values in Silva and Castro were considerably 
METER WITHOUT RHYTHMIC PATTERN REPETITIONS INCREASES PRE-ATTENTIVE PROCESSING

lower than in the present study. Concerning the present results on surface meter (attentive only), they are in line with those from Geiser et al. (2009), but at odds with several other studies that found pre-attentive processing in surface meter (Bouwer et al., 2014, 2016; Vuust et al., 2009; Winkler et al., 2009; Zhao et al., 2017). The reason for this divergence may be that several of these studies used sound omissions instead of sound additions as markers of deviance (Bower et al., 2014, 2019; Winkler, 2009). This may have induced different processes, namely something closer to meter as temporal orientation, guiding listeners attention to the presence vs. absence of a target event. The similarity with Geiser et al's (2009) results makes sense given the identical paradigms, based on sound additions creating meter deviants. Future research could compare the two types of surface meter deviances - omissions vs. additions - to test whether using sound omissions increases pre-attentive processing due to the induction of processes related to meter as temporal orientation.

We interpreted the increased presence of pre-attentive processing in structural meter compared to surface meter as an indicator that increased cognitive load (here, increased abstraction) recruits pre-attentive processing. Although this reading is consistent with other pieces of evidence - increased pre-attentive processing in dual-task contexts, poorer behavioral performance in structural meter, different patterns of attention in low vs. high d-prime participants, more marked pre-attentive processing in Silva and Castro (2019) going along with poorer behavioral performance - we cannot exclude alternative explanations. One that pops out from behavioral results is the possibility that surface meter captures participants' attention more strongly, even when they are asked not to pay attention to temporal information: we saw increased pitch discrimination in surface than in structural meter, raising the possibility that participants were less able to ignore temporal information in surface than in structural meter. 
METER WITHOUT RHYTHMIC PATTERN REPETITIONS INCREASES PRE-ATTENTIVE PROCESSING

Could this mean that the increased pre-attentive processing we saw in structural meter was due to participants' increased ability to ignore meter, rather than to the increased cognitive load imposed by structural meter? One argument against this possibility is that, despite the poorer pitch discrimination in surface meter, participants still performed well above chance. Therefore, they were able to focus their attention on pitch. Moreover, we can look at differences in pitch discrimination as additional evidence for our main hypothesis: structural meter imposes greater cognitive demand, it is preferably processed pre-attentively, and thus it frees attentive mechanisms to focus on the pitch deviant detection task. Testing the "easier to ignore" vs. the "harder to process" hypotheses on structural meter remains a challenge for future research.

Our findings raise at least three additional research questions for the future. One relates to direct comparisons between meter processing for temporal orientation and meter processing for temporal expectation as far as attention is concerned: is pre-attentive processing increased in the former? A second question concerns the duality structure-surface applied to beat: does the processing of structural beat, i.e., an underlying beat, hidden under a varying surface, also elicits increased pre-attentive-processing compared to surface beat, i.e., isochronous events? Finally, a major question that emerges is whether the beat level, due to its lower position in the temporal hierarchy (Fitch, 2013) and its expected lower complexity (Arcadi, Robert \& Mugurusi, 2004; Donnet et al., 2014; Fitch, 2015; Merchant et al., 2015; Nave-Blodgett et al., 2020b; Thaut et al., 2014; Winkler et al., 2009; Zarco et al., 2009), requires less pre-attentive processing than meter. Here, it could be important to distinguish between beat nested into meter (presence of meter cues, effective hierarchy) and beat per se (lack of meter cues, no apparent hierarchy). 
METER WITHOUT RHYTHMIC PATTERN REPETITIONS INCREASES PRE-ATTENTIVE PROCESSING

\section{Conclusion}

Our study contributed to challenge current frameworks by supporting a novel hypothesis concerning the engagement of attention in meter processing: attentive processing may be less efficient as abstraction increases. Also, we provided pioneer evidence that processing meter with the aid of rhythmic pattern recognition - the dominant framework in current research - is not equivalent to processing meter without that aid. In this sense, our findings promote increased awareness in future research when it comes to measure meter processing.

\section{Conflict of interest}

The authors declare that the research was conducted in the absence of any commercial or financial relationships that could be construed as a potential conflict of interest.

Authors' contributions

Hidden

Funding

Hidden

\section{Acknowledgements}

Hidden

\section{Data Availability Statement}

The datasets generated for this study and the outcomes of analyses can be found in the OSF link: https://osf.io/94j5v/?view_only=e5d35905163e4403b94d28a82316d679 
METER WITHOUT RHYTHMIC PATTERN REPETITIONS INCREASES PRE-ATTENTIVE PROCESSING

\section{References}

Arcadi, A. C., Robert, D., \& Mugurusi, F. (2004). A comparison of buttress drumming by male chimpanzees from two populations. Primates, 45(2), 135-139. doi: 10.1007/s10329003-0070-8

Bégel, V., Benoit, C., Correa, A., Cutanda, D., Kotz, S., \& Dalla Bella, S. (2017). "Lost in time” but still moving to the beat. Neuropsychologia, 94, 129-138. doi:

10.1016/j.neuropsychologia.2016.11.022

Biel, A. L., \& Friedrich, E. V. C. (2018). Why you should report Bayes factors in your transcranial brain stimulation studies. Frontiers in Psychology, 9. https://doi.org/10.3389/fpsyg.2018.01125

Bolger, D., Trost, W., \& Schön, D. (2013). Rhythm implicitly affects temporal orienting of attention across modalities. Acta Psychologica, 142(2), 238-244. https://doi.org/10.1016/j.actpsy.2012.11.012

Bolger, D., Coull, J. T., \& Schön, D. (2014). Metrical rhythm implicitly orients attention in time as indexed by improved target detection and left inferior parietal activation. Journal of Cognitive Neuroscience, 26(3), 593-605. https://doi.org/10.1162/jocn_a_00511

Bouwer, F. L., Werner, C. M., Knetemann, M., \& Honing, H. (2016). Disentangling beat perception from sequential learning and examining the influence of attention and musical abilities on ERP responses to rhythm. Neuropsychologia, 85, 80-90. https://doi.org/10.1016/j.neuropsychologia.2016.02.018

Bouwer, F. L., Zuijen, T. L. V., \& Honing, H. (2014). Beat processing is pre-attentive for metrically simple rhythms with clear accents: An ERP Study. PLOS ONE, 9(5), e97467. https://doi.org/10.1371/journal.pone.0097467 
METER WITHOUT RHYTHMIC PATTERN REPETITIONS INCREASES PRE-ATTENTIVE PROCESSING

Bouwer, F., \& Honing, H. (2015). Temporal attending and prediction influence the perception of metrical rhythm: evidence from reaction times and ERPs. Frontiers in Psychology, 6. doi: 10.3389/fpsyg.2015.01094

Celma-Miralles, A., \& Toro, J. M. (2019). Ternary meter from spatial sounds: Differences in neural entrainment between musicians and non-musicians. Brain and Cognition, 136, 103594.

Celma-Miralles, A., de Menezes, R., \& Toro, J. (2016). Look at the beat, feel the meter: Topdown effects of meter induction on auditory and visual modalities. Frontiers in Human Neuroscience, 10. doi: 10.3389/fnhum.2016.00108

Colley, I. D., Keller, P. E., \& Halpern, A. R. (2018). Working memory and auditory imagery predict sensorimotor synchronization with expressively timed music. Quarterly Journal of Experimental Psychology, 71(8), 1781-1796. https://doi.org/10.1080/17470218.2017.1366531

Donnet, S., Bartolo, R., Fernandes, J., Cunha, J., Prado, L., \& Merchant, H. (2014). Monkeys time their pauses of movement and not their movement-kinematics during a synchronization-continuation rhythmic task. Journal of Neurophysiology, 111(10), 2138-2149. doi: 10.1152/jn.00802.2013

Einarson, K., \& Trainor, L. (2016). Hearing the beat. Music Perception, 34(1), 56-70. doi: 10.1525/mp.2016.34.1.56

Escoffier, N., Sheng, D. Y. J., \& Schirmer, A. (2010). Unattended musical beats enhance visual processing. Acta Psychologica, 135(1), 12-16. https://doi.org/10.1016/j.actpsy.2010.04.005 
METER WITHOUT RHYTHMIC PATTERN REPETITIONS INCREASES PRE-ATTENTIVE PROCESSING

Faul, F., Erdfelder, E., Lang, A.-G., \& Buchner, A. (2007). G*Power 3: A flexible statistical power analysis program for the social, behavioral, and biomedical sciences. Behavior Research Methods, 39(2), 175-191. https://doi.org/10.3758/BF03193146

Fitch, W. (2013). Rhythmic cognition in humans and animals: distinguishing meter and pulse perception. Frontiers In Systems Neuroscience, 7. doi: 10.3389/fnsys.2013.00068

Fitch, W. (2015). Four principles of bio-musicology. Philosophical Transactions of the Royal Society B: Biological Sciences, 370(1664), 20140091. doi: 10.1098/rstb.2014.0091

Fitch, W. (2016). Dance, music, meter and groove: A forgotten partnership. Frontiers in Human Neuroscience, 10. doi: 10.3389/fnhum.2016.00064

Friederici, A. D. (2002). Towards a neural basis of auditory sentence processing. Trends in Cognitive Sciences, 6(2), 78-84. https://doi.org/10.1016/S1364-6613(00)01839-8

Geiser, E., Sandmann, P., Jäncke, L., \& Meyer, M. (2010). Refinement of metre perception training increases hierarchical metre processing. European Journal of Neuroscience, 32(11), 1979-1985. doi: 10.1111/j.1460-9568.2010.07462.x

Geiser, E., Ziegler, E., Jancke, L., \& Meyer, M. (2009). Early electrophysiological correlates of meter and rhythm processing in music perception. Cortex, 45(1), 93-102. doi: 10.1016/j.cortex.2007.09.010

Grahn, J. A. (2012). Neural mechanisms of rhythm perception: Current findings and future perspectives. Topics in Cognitive Science, 4(4), 585-606. https://doi.org/10.1111/j.17568765.2012.01213.x

Hagoort, P., \& Brown, C. M. (2000). ERP effects of listening to speech compared to reading: The P600/SPS to syntactic violations in spoken sentences and rapid serial visual 
METER WITHOUT RHYTHMIC PATTERN REPETITIONS INCREASES PRE-ATTENTIVE PROCESSING

presentation. Neuropsychologia, 38(11), 1531-1549. https://doi.org/10.1016/S00283932(00)00053-1

Hassin, R. R., Bargh, J. A., Engell, A. D., \& McCulloch, K. C. (2009). Implicit working memory. Consciousness and Cognition, 18(3), 665-678. https://doi.org/10.1016/j.concog.2009.04.003

JASP Team (2020). JASP (Version 0.14.1)[Computer software].

Keller, P. E., \& Burnham, D. K. (2005). Musical meter in attention to multipart rhythm. Music Perception, 22(4), 629-661. https://doi.org/10.1525/mp.2005.22.4.629

Kotz, S., Ravignani, A., \& Fitch, W. (2018). The evolution of rhythm processing. Trends In Cognitive Sciences, 22(10), 896-910. doi: 10.1016/j.tics.2018.08.002

Lenc, T., Keller, P., Varlet, M., \& Nozaradan, S. (2020). Attention affects overall gain but not selective contrast at meter frequencies in the neural processing of rhythm. bioRxiv. $10.1101 / 2020.09 .23 .309443$

Masters, R. S. W. (1992). Knowledge, knerves and know-how: The role of explicit versus implicit knowledge in the breakdown of a complex motor skill under pressure. British Journal of Psychology, 83(3), 343-358. https://doi.org/10.1111/j.20448295.1992.tb02446.x

Merchant, H., Grahn, J., Trainor, L., Rohrmeier, M., \& Fitch, W. (2015). Finding the beat: a neural perspective across humans and non-human primates. Philosophical Transactions of the Royal Society B: Biological Sciences, 370(1664), 20140093. doi:

$10.1098 /$ rstb.2014.0093 
METER WITHOUT RHYTHMIC PATTERN REPETITIONS INCREASES PRE-ATTENTIVE PROCESSING

Nave-Blodgett, J. E., Snyder, J. S., \& Hannon, E. (2020a). Auditory superiority for perceiving the beat level but not the measure level in music. PsyArXiv. https://doi.org/10.31234/osf.io/ch7ds

Nave-Blodgett, J., Snyder, J., \& Hannon, E. (2020b). Hierarchical beat perception develops throughout childhood and adolescence and is enhanced in those with musical training. Journal of Experimental Psychology: General. doi: 10.1037/xge0000903

Neuhaus, C., \& Knösche, T. R. (2006). Processing of rhythmic and melodic Gestalts: An ERP Study. Music Perception, 24(2), 209-222. https://doi.org/10.1525/mp.2006.24.2.209

Oostenveld, R., Fries, P., Maris, E., \& Schoffelen, J. (2011). FieldTrip: Open source software for advanced analysis of MEG, EEG, and invasive electrophysiological data.

Computational Intelligence And Neuroscience, 2011, 1-9. doi: 10.1155/2011/156869

Palmer, C., \& Krumhansl, C. L. (1990). Mental representations for musical meter. Journal of Experimental Psychology: Human Perception and Performance, 16(4), 728-741. https://doi.org/10.1037/0096-1523.16.4.728

Perna, F., Pavani, F., Zampini, M., \& Mazza, V. (2018). Behavioral dynamics of rhythm and meter perception: The effect of musical expertise in deviance detection. Timing \& Time Perception, 6(1), 32-53. doi: 10.1163/22134468-00002100

Reber, A. S., Kassin, S. M., Lewis, S., \& Cantor, G. (1980). On the relationship between implicit and explicit modes in the learning of a complex rule structure. Journal of Experimental Psychology: Human Learning and Memory, 6(5), 492-502. https://doi.org/10.1037/02787393.6.5.492 
METER WITHOUT RHYTHMIC PATTERN REPETITIONS INCREASES PRE-ATTENTIVE PROCESSING

Schwartze, M., Rothermich, K., Schmidt-Kassow, M., \& Kotz, S. A. (2011). Temporal regularity effects on pre-attentive and attentive processing of deviance. Biological Psychology, 87(1), 146-151. https://doi.org/10.1016/j.biopsycho.2011.02.021

Shine, J., \& Shine, R. (2014). Delegation to automaticity: The driving force for cognitive evolution? Frontiers in Neuroscience, 8. https://doi.org/10.3389/fnins.2014.00090

Sidiras, C., Iliadou, V. V., Nimatoudis, I., Grube, M., Griffiths, T., \& Bamiou, D.-E. (2019). Deficits in auditory rhythm perception in children with auditory processing disorder are unrelated to attention. Frontiers in Neuroscience, 13. https://doi.org/10.3389/fnins.2019.00953

Silva, S., \& Castro, S. (2016). Moving stimuli facilitate synchronization but not temporal perception. Frontiers in Psychology, 7. doi: 10.3389/fpsyg.2016.01798

Silva, S., \& Castro, S. (2018). Meter for what? Meter strength affects ordinal but not temporal learning. Cogent Psychology, 5(1), 1537062. doi: 10.1080/23311908.2018.1537062

Silva, S., \& Castro, S. (2019). Structural meter perception is pre-attentive. Neuropsychologia, 133, 107184. doi: 10.1016/j.neuropsychologia.2019.107184

Stanislaw, H., \& Todorov, N. (1999). Calculation of signal detection theory measures. Behavior Research Methods, Instruments, \& Computers, 31(1), 137-149. https://doi.org/10.3758/BF03207704

Thaut, M., Trimarchi, P., \& Parsons, L. (2014). Human brain basis of musical rhythm perception: Common and distinct neural substrates for meter, tempo, and pattern. Brain Sciences, 4(2), 428-452. doi: 10.3390/brainsci4020428

van Doorn, J., van den Bergh, D., Böhm, U., Dablander, F., Derks, K., Draws, T., Etz, A., Evans, N. J., Gronau, Q. F., Haaf, J. M., Hinne, M., Kucharský, Š., Ly, A., Marsman, M., 
METER WITHOUT RHYTHMIC PATTERN REPETITIONS INCREASES PRE-ATTENTIVE PROCESSING

Matzke, D., Gupta, A. R. K. N., Sarafoglou, A., Stefan, A., Voelkel, J. G., \& Wagenmakers, E.-J. (2020). The JASP guidelines for conducting and reporting a Bayesian analysis. Psychonomic Bulletin \& Review. https://doi.org/10.3758/s13423-02001798-5

Vuust, P., Ostergaard, L., Pallesen, K., Bailey, C., \& Roepstorff, A. (2009). Predictive coding of music - Brain responses to rhythmic incongruity. Cortex, 45(1), 80-92. doi: 10.1016/j.cortex.2008.05.014

Westfall, J. (2016). PANGEA: Power ANalysis for GEneral Anova designs. Retrieved from http://jakewestfall.org/publications/pangea.pdf

Windsor, W. L. (1993). Dynamic accents and the categorical perception of metre. Psychology of Music, 21(2), 127-140. doi: 10.1177/030573569302100203

Winkler, I., Denham, S., \& Escera, C. (2013). Auditory Event-related Potentials. Em D. Jaeger \& R. Jung (Eds.), Encyclopedia of Computational Neuroscience (pp. 1-29). Springer. https://doi.org/10.1007/978-1-4614-7320-6_99-1

Winkler, I., Haden, G., Ladinig, O., Sziller, I., \& Honing, H. (2009). Newborn infants detect the beat in music. Proceedings of the National Academy of Sciences, 106(7), 2468-2471. doi: 10.1073/pnas.0809035106

Yates, C., Justus, T., Atalay, N., Mert, N., \& Trehub, S. (2016). Effects of musical training and culture on meter perception. Psychology of Music, 45(2), 231-245. doi: $10.1177 / 0305735616657407$

Zarco, W., Merchant, H., Prado, L., \& Mendez, J. (2009). Subsecond timing in primates: Comparison of interval production between human subjects and Rhesus monkeys. Journal of Neurophysiology, 102(6), 3191-3202. doi: 10.1152/jn.00066.2009 
METER WITHOUT RHYTHMIC PATTERN REPETITIONS INCREASES PRE-ATTENTIVE PROCESSING

Zhao, T., Lam, H., Sohi, H., \& Kuhl, P. (2017). Neural processing of musical meter in musicians and non-musicians. Neuropsychologia, 106, 289-297. doi: 10.1016/j.neuropsychologia.2017.10.007 
METER WITHOUT RHYTHMIC PATTERN REPETITIONS INCREASES PRE-ATTENTIVE PROCESSING

Appendix A: Standard stimuli (left - standards; right - surface meter standards)

\begin{tabular}{|c|c|}
\hline 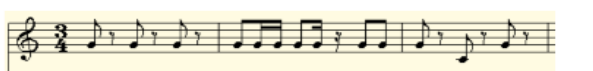 & 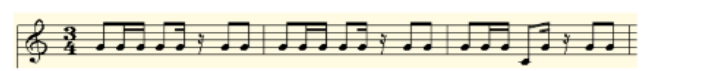 \\
\hline 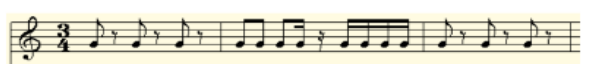 & (马) \\
\hline 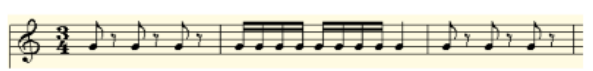 & 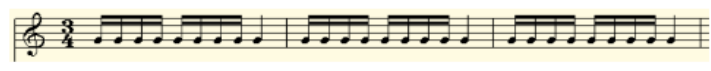 \\
\hline 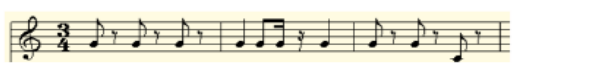 & 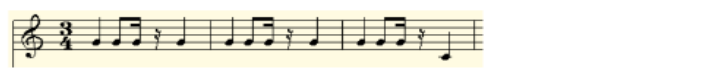 \\
\hline 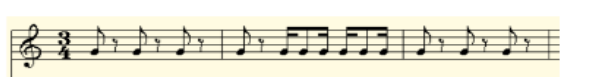 & 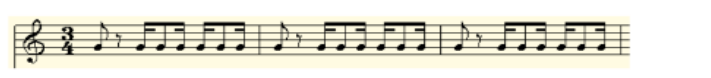 \\
\hline 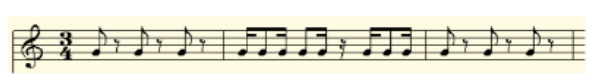 & 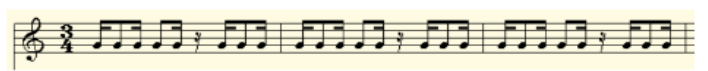 \\
\hline 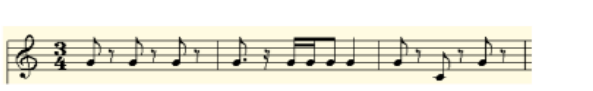 & 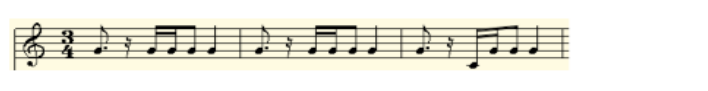 \\
\hline 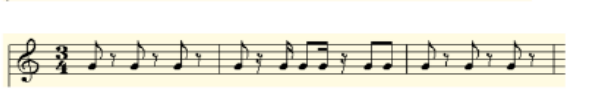 & 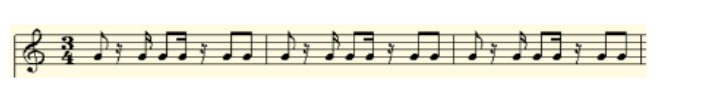 \\
\hline 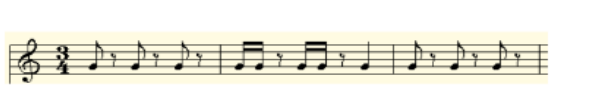 & 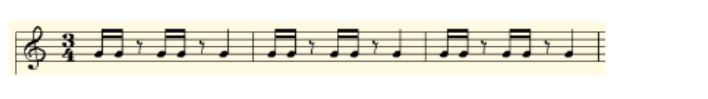 \\
\hline 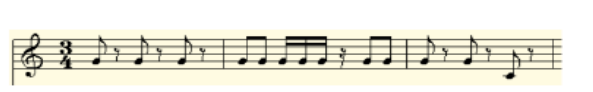 & 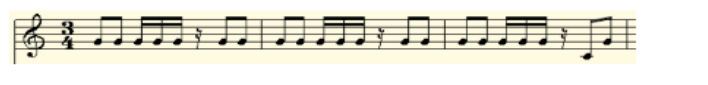 \\
\hline 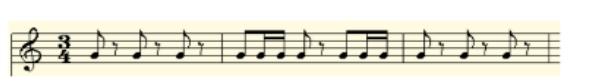 & ל : \\
\hline 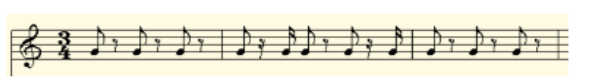 & 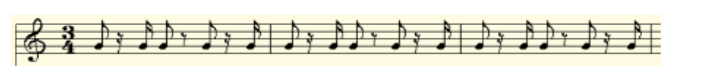 \\
\hline 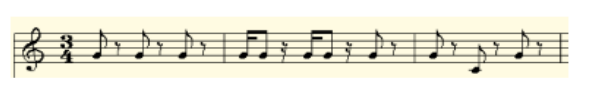 & 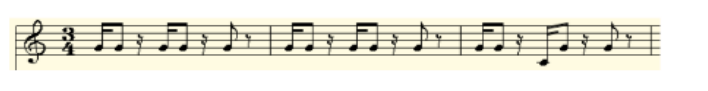 \\
\hline 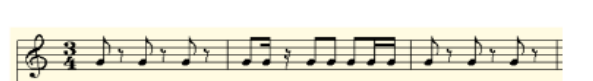 & 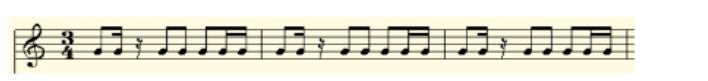 \\
\hline
\end{tabular}


METER WITHOUT RHYTHMIC PATTERN REPETITIONS INCREASES PRE-ATTENTIVE PROCESSING

\begin{tabular}{|c|c|}
\hline 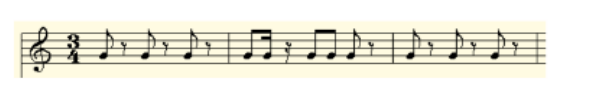 & 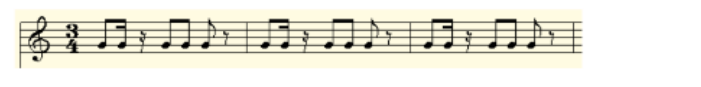 \\
\hline 32110.1110 & 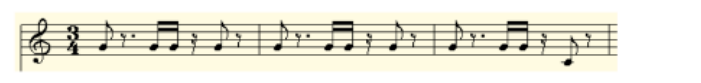 \\
\hline 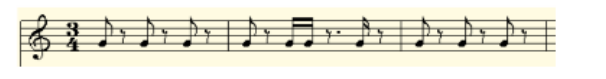 & 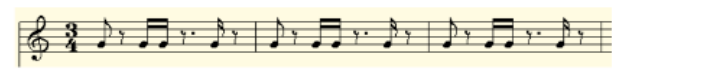 \\
\hline $3+1.1 .19$ & 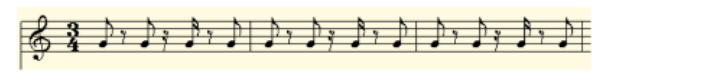 \\
\hline $3+7.7+5$ & $b .1 ., 1,5, \ldots, \ldots$ \\
\hline 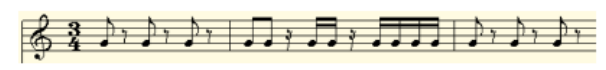 & 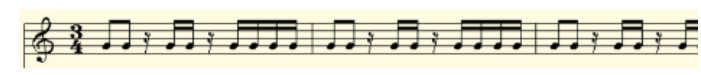 \\
\hline 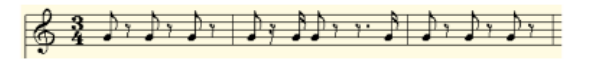 & 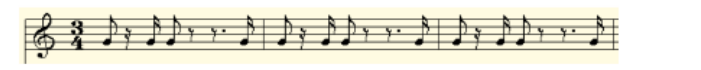 \\
\hline 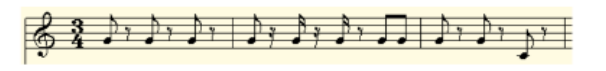 & 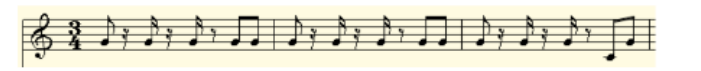 \\
\hline 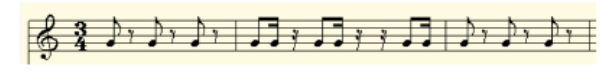 & 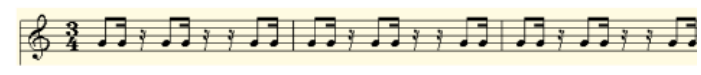 \\
\hline 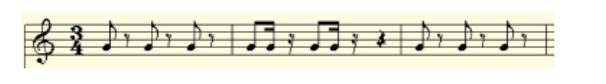 & 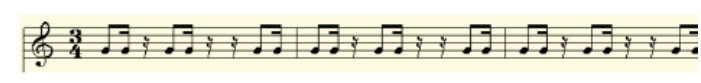 \\
\hline 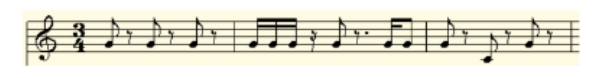 & 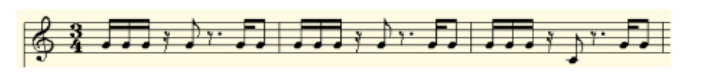 \\
\hline 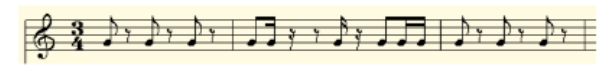 & 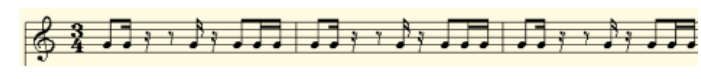 \\
\hline $3+1.010 .19$ & 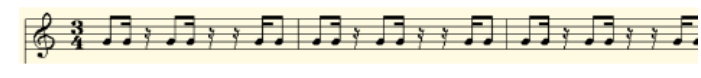 \\
\hline 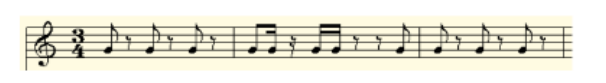 & 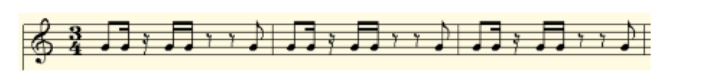 \\
\hline $3+1,3+1,10$ & 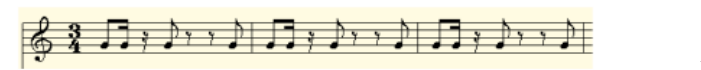 \\
\hline
\end{tabular}


METER WITHOUT RHYTHMIC PATTERN REPETITIONS INCREASES PRE-ATTENTIVE PROCESSING

\section{Appendix B}

Comparison between analysis for all trials vs. analysis for correct trials only

Table E1. Summary of the analysis for all trials. Are there differences in attention requirements?

\section{Meter}

No. Attention-independent processing

No. Attention-independent processing

P3

P600
No.

Yes. Attentive only more pronounced P600 for deviants.

\section{Surface meter}

Yes. Attentive only more pronounced P1 for deviants.

Yes. Attentive only attenuated P2 for deviants.

Yes. More pronounced P3 in attended conditions.

Yes. Attentive only more pronounced P600 for deviants.

Table E2. Summary of the analysis considering correct trials only. Are there differences in attention requirements?

\section{Meter}

P1

P2

P3

P600
No. Attention-independent

processing

No. Attention-independent

processing

No.

Yes. Attentive only more pronounced P600 for deviants.

\section{Surface meter}

Yes. Attentive only more pronounced P1 for deviants.

Yes. Attentive only attenuated P2 for deviants.

Yes. More pronounced P3 in attended conditions. (Bayesian only)

Yes. Attentive only more pronounced P600 for deviants.

Note. In general, the results are similar to the main analysis. 
METER WITHOUT RHYTHMIC PATTERN REPETITIONS INCREASES PRE-ATTENTIVE PROCESSING

\section{Appendix C}

EEG correlates of structural meter processing following analysis by $300 \mathrm{~ms}$ time windows. Waveforms are plotted for the Right Central region electrodes.
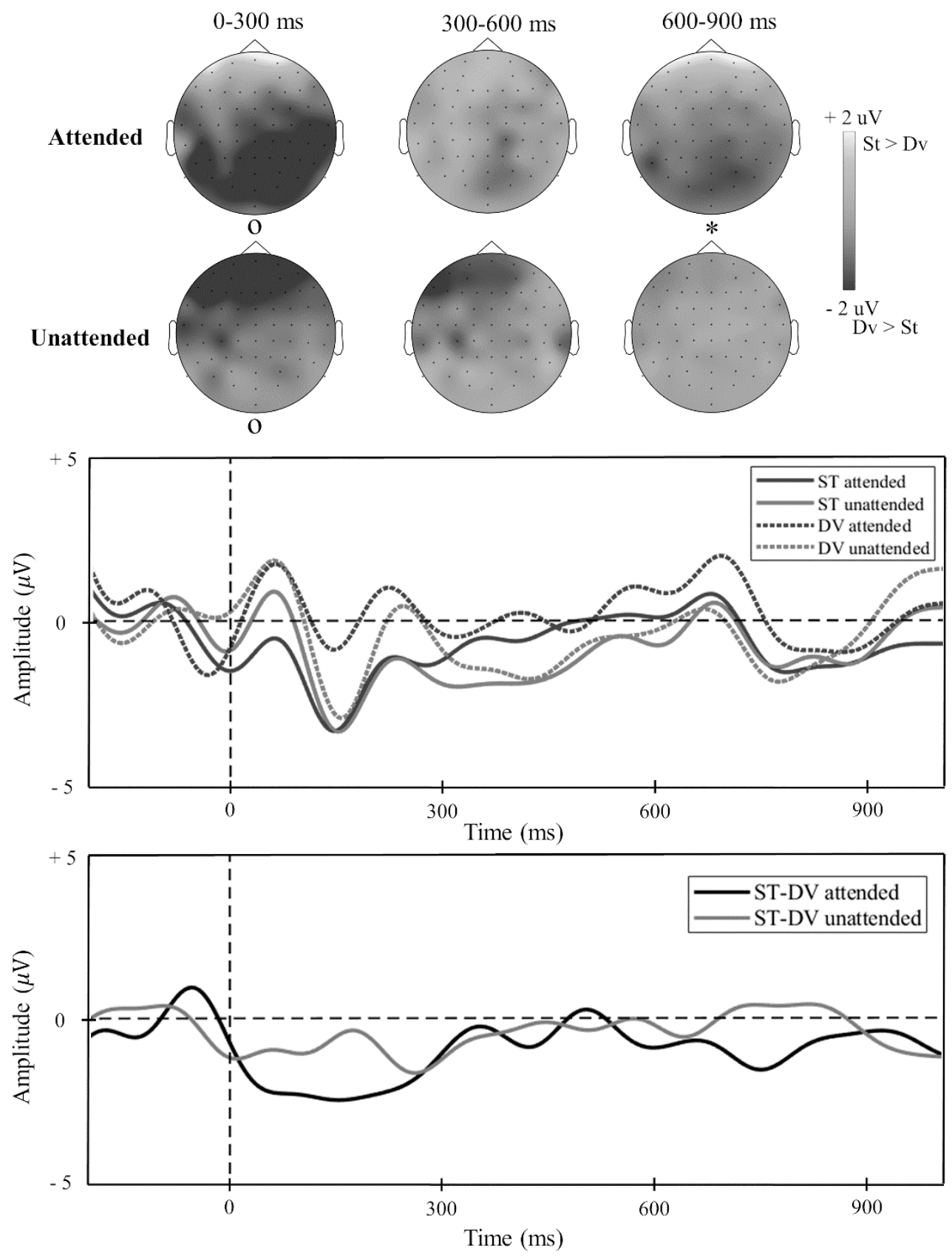


\section{Appendix D}

EEG correlates of surface meter processing following analysis by $300 \mathrm{~ms}$ time windows. Waveforms are plotted for the Middle Posterior region electrodes.
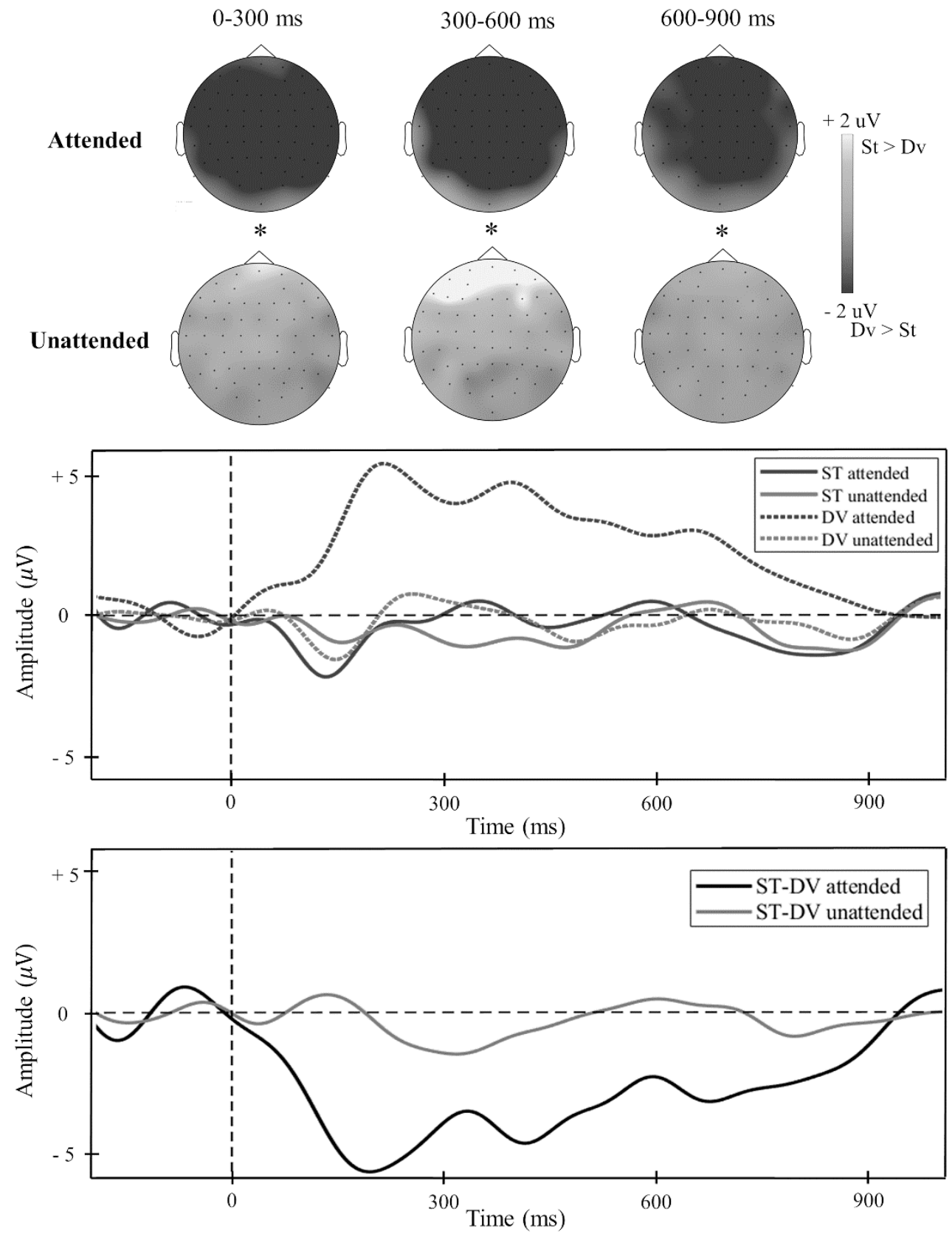